\title{
Psychoeducation: perspectives from individuals on sick leave who are at risk of having a mental disorder
}

\author{
Line Hille Højfeldt ${ }^{\star}$ \\ Psychiatric Research Unit West, Regional Psychiatric Services West, \\ Central Denmark Region, Herning, Denmark \\ Email: linehille@ hotmail.com \\ *corresponding author
}

\section{Pernille Pedersen}

Psychiatric Research Unit West, Regional Psychiatric Services West, Central Denmark Region, Herning, Denmark

Institute of Clinical Medicine, University of Aarhus, Aarhus, Denmark

Public Health and Quality Improvement, Central Denmark Region, Aarhus, Denmark

Email: Pernille.Pedersen@stab.rm.dk

\section{Kirsten Schultz Petersen}

CFK - Public Health and Quality Improvement, Central Denmark Region, Aarhus, Denmark

Department of Social Medicine and Rehabilitation, Faculty of Health Sciences, University of Aarhus, Denmark

Department of Health Sciences and Technology, Aalborg University, Aalborg, Denmark

Email: ksp@hst.aau.dk

\section{Lars Peter Andersen}

Danish Ramazzini Centre, Department of Occupational Medicine, The Regional Hospital West Jutland - University Research Clinic, Herning, Denmark

Email: Lars.Peter.Soenderbo.Andersen@vest.rm.dk 


\begin{abstract}
The large number of people on sickness and disability benefits due to mental disorders in Denmark has increased the need for improved rehabilitative services to facilitate their return to work. The aim of the present study was to explore the use of psychoeducation in relation to the standard services of a Danish job centre for individuals on sick leave with regard to relevance, elements contributing to recovery, and improvements of psychoeducation as an intervention. Semi-structured interviews were conducted with 16 individuals on sick leave who were at risk of having a mental disorder. The interviews were analysed using systematic text condensation inspired by Giorgi's phenomenological analysis. The resulting core themes describing psychoeducation with respect to the standard services of the job centre included access and relevance of psychoeducation in relation to the job centre's standard services, social support, self-care, and psychoeducation intervention. This study concludes that the informants consider psychoeducation a relevant offer to individuals on sick leave who are at risk of having a mental disorder. The relevance of the standard services of the job-centre services was considered to be low. Furthermore, psychoeducation reinforces peer support and inclusion of relatives as elements to aid recovery to a much larger extent than do the standard services of the job centre. In general, the results support the use of psychoeducation in a municipal job-centre setting as a targeted and beneficial offer to individuals on sick leave who are at risk of having a mental disorder.
\end{abstract}

Keywords: Qualitative, psychoeducation, job centre, sick leave, mental health, return to work, rehabilitation, social relations, peer support

\title{
Introduction
}

Common mental disorders constitute the most prevalent causes of long-term sickness absence (more than eight weeks) and are the reason for 50 per cent of all disability pensions given in 2011 in Denmark (Danish Employers' Confederation, 2014). This has increased the need for improved rehabilitative services to enable individuals on sick leave with mental disorders to return to work. In recent years, the focus has been on developing rehabilitative interventions that facilitate a return to work for these individuals. A broad range of interventions have been quantitatively tested but have not yet been implemented and applied in the practical rehabilitation process aimed at the return to work (Arends et al., 2012; Nieuwenhuijsen et al., 2008). Qualitative studies have described a range of barriers for people with mental-health problems in these rehabilitative processes, such as acceptance from the surroundings, recognition of mental-health problems, individual factors and attitudes, contact, trust and support regarding the workplace and professionals (Muijzer, Brouwer, Geertzen, \& Groothoff, 2012; Noordik, 2011; Ståhl \& Edvardsson Stiwne, 2014). Rehabilitative processes are, however, complex, involving a range of actors and efforts (Rouget \& Aubry, 2007). In a Danish context, we have little knowledge of the practical rehabilitative efforts facilitating the return to work from the perspective of individuals on sick leave who are at risk of having a mental disorder. 
Danish municipal case-management centres (job centres) administrate sickness-benefit cases and are responsible for the process of returning individuals on sick leave back to work. General practitioners and health-care services are responsible for the diagnosis and treatment of the conditions responsible for sickness absence. Job centres provide activation programmes to individuals on sick leave, programmes which can be considered the core services of the job centre. A critique of these services emphasizes the lack of evidence-based return-to-work interventions, and that the services are not necessarily targeted at individuals on sick leave who are at risk of having a mental disorder (Stafford \& Colom, 2013). Hillborg (2010) has found that individuals on sick leave due to mental disorders may experience despondency and setbacks if they do not perceive the rehabilitation services as being relevant. A recent meta-synthesis of studies on return to work among employees with mental-health problems (Andersen, Nielsen, \& Brinkmann, 2012) shows that employees of individuals on sick leave mainly had negative experiences with the social-insurance offices. They felt that the socialinsurance office did not provide the necessary support because there was no focus on their mental-health problem or needs. Therefore, this study aims to explore sick-listed individuals' experiences with psychoeducation in relation to the standard services of a job centre.

Psychoeducation is an intervention that targets individuals with mental disorders, providing them with tools to support their involvement in recovery and coping (Donker, Griffiths, Cuijpers, \& Christensen, 2009; Stafford \& Colom, 2013). Psychoeducation includes diverse psychotherapeutic techniques, for example, cognitive behavioural and problem-solving therapy (Casañas et al., 2012), which promote awareness and forethoughtfulness in relation to the recognition of episodic recurrences, and improve psychosocial and occupational functioning and quality of life (Colom, 2011). Quantitative studies have examined psychoeducation in diverse groups with mental-health problems (Colom et al., 2009; Morokuma et al., 2013; Rouget \& Aubry, 2007), but few qualitative studies have explored participants' perspectives of participating in psychoeducation. Psychoeducation has been conducted among patients with schizophrenia and patients suffering from bipolar affective disorder (Aho-Mustonen, Miettinen, \& Räty, 2010; O'Connor, Gordon, Graham, Kelly, \& O'Grady-Walshe, 2008). The experiences of the participants of these studies were positive regarding the information about their illness and the opportunities to discuss illness-related issues. The psychoeducation intervention appears to have positive effects on activation and social interaction. Psychoeducation also seemed to have a potential to change participants' perception and acceptance of their illness, which enabled them to cope better with their illness.

To our knowledge, psychoeducation has not previously been used as a rehabilitative return-to-work intervention in a municipal job-centre setting for individuals on sick leave who are at risk of a having a mental disorder. This study provides insight into personal experiences with psychoeducation as a rehabilitative intervention for the return to work as a part of a job centre's standard services.

\section{Aim of study}

The aim of the study has been to explore the experiences of individuals on sick leave who are at risk of having a mental disorder from the perspective of 
individuals receiving the psychoeducation and individuals receiving standard services from the job centres. This study has focused on three issues: 1) the relevance of psychoeducation in relation to job centres' standard services; 2) the identification of elements in psychoeducation in relation to job centres' standard services that might contribute to recovery; and 3) the improvements of psychoeducation as a rehabilitative intervention aimed at a return to work.

\section{Materials and Methods}

\section{Participants}

The first author conducted 16 qualitative interviews with individuals attending another study involving a randomized control trial (RCT) testing the effect of psychoeducation on the return to work among 430 individuals on sick leave as a supplement to standard job-centre services. The RCT study was conducted in four Danish municipalities from September 2012 to April 2014 (Pedersen et al., 2014). The participants included in the research project were of working age, on sick leave from part-time or full-time work or unemployment and scored five or less on Symptom Checklist-8 Anxiety Depression (SCL-8AD), which is a screening instrument for detecting mental disorders among individuals on sick leave (Søgaard \& Bech, 2009). All participants were obliged to participate in interviews with a job-centre social worker and, based on individual assessments, were entitled to receive individual, standard job-centre services such as mindfulness, physical exercise, a slow return to work, job training or special care, and short lectures. The psychoeducational sessions were not part of the standard services but were carried out at the participating job centres. The informants in the present study (eight women and eight men, median age 47 years (range 29-60) were selected with the aim of assuring a variation in gender, age, work status, and level of education. Eight informants had received the standard services of the job centre (control group (C)), and eight informants received the standard services of the job centre plus psychoeducation (intervention group (I)). Characteristics of the 16 informants are presented in Table 1. Eight of the informants were still employed or under education during the interview, two were uncertain about their present work status because they had lost contact with their employer, and six were unemployed. At the time of the interview, six informants had fully returned to work, and ten were on full-time sick leave. Depression was the most common self-reported reason for sick leave; the other reasons were stress, anxiety, and physical problems. The informants had been on sick leave between three months and one year.

\section{Psychoeducation sessions}

The psychoeducation intervention in this study was designed as group-based sessions, two hours once a week for six weeks. It was a slow-open group, where new participants could join the sessions shortly after they had agreed to participate. A social worker, psychiatric nurses, a physiotherapist, a psychologist, and a person previously on sick leave due to mental-health problems led the sessions. The sessions focused on stress and working life and were a mix of didactic lectures and group discussions. The purpose of the psychoeducation intervention was to provide individuals with the capacity to understand and improve their own situation through knowledge, dialogue, 
personal experiences, tools, problem-solving techniques, and coping strategies. The six sessions (a-f) dealt with the following themes: (a) information on symptoms; (b) information on options and coping strategies; (c) counselling on the basis of sick-leave legislation; (d) information on mentalhealth psychology; (e) physical exercise; (f) a presentation of a personal story from a woman previously on sick leave due to mental-health problems and a discussion of relational support for the individual on sick leave. The last session ( $f$ ) included the participants' relatives, with the purpose of providing them with information and tools to support the individual on sick leave (Pedersen et al., 2014).

Table 1: Characteristics of the informants

\begin{tabular}{llllll} 
Gender & Age & Work status & Educational level & $\begin{array}{l}\text { Intervention } \\
\text { /Control }\end{array}$ & $\begin{array}{l}\text { Status of sick } \\
\text { leave during } \\
\text { interview }\end{array}$ \\
\hline F & 51 & Unemployed & Medium & I & On sick leave \\
F & 55 & Employed & Skilled/trained & I & Full-time work \\
M & 44 & Don't know & Skilled/trained & I & On sick leave \\
F & 42 & Employed & None & I & On sick leave \\
M & 48 & Unemployed & None & I & On sick leave \\
M & 38 & Unemployed & Skilled/trained & I & On sick leave \\
M & 52 & Unemployed & Skilled/trained & I & On sick leave \\
F & 41 & Employed & Medium & I & Full-time work \\
F & 47 & Employed & Medium & C & Full-time work \\
M & 54 & Employed & None & C & On sick leave \\
F & 55 & Employed & None & C & Full-time work \\
F & 52 & Employed & Medium & C & Full-time work \\
M & 40 & Unemployed & Skilled/trained & C & On sick leave \\
M & 60 & Don't know & Skilled/trained & C & On sick leave \\
F & 29 & Under education & Skilled/trained & C & Full-time \\
& & & & & education \\
M & 47 & Unemployed & None & C & On sick leave
\end{tabular}

\section{Data collection}

This study had a descriptive, explorative approach in order to enable each informant to report individual (positive and negative) experiences regarding psychoeducation as an addition to the job centres' standard services within about a month after having received the psychoeducational sessions. Contact with informants was made by telephone. We presented the aim, purpose, expected duration of the interviews, and a choice of conducting the interview in the job-centre offices, in the informants' own home or at the research office. We developed a semi-structured interview guide with open-ended questions, which were based on the themes of rehabilitation. As the interview guide (see Table 2) indicates, the themes of rehabilitation contained principles for an 
optimal intervention in individuals with limitations, and covered issues such as relevance in relation to a specific health problem, time, involvement of relatives, and quality of services (Marselisborgcentret \& Rehabiliteringsforum Danmark, 2004). All interviews were conducted by the first author and began with background and introductory questions about their sick-leave history. All interviews were audio recorded and lasted between 19 minutes and one hour and 23 minutes. The various durations were due to the informants' individual sick-leave histories, the number of standard services received at different job centres, and whether they had participated in the psychoeducation sessions.

\section{Data analysis}

The interviews were transcribed, coded in N-Vivo10, and analysed inductively. This analysis used systematic text condensation inspired by Giorgi's phenomenological analysis in four stages: (1) forming an overview of themes by listening to the interviews multiple times; (2) coding and identifying units of meaning, representing subthemes of different aspects of the informants' experiences; (3) condensing and summarizing the contents of each of the individual meaning units within the subthemes; and (4) summarizing the contents of each meaning unit in order to generalize descriptions and patterns of their experiences (Malterud, 2011). The phenomenological analysis identified four themes and six subthemes reflecting the informants' experience by focusing on the different perspectives on psychoeducation and the job centres' standard services.

\section{Table 2: Semi-structured interview guide}

Introduction: Why are you/have you been on sick leave?

1 Can you describe the standard services you received from the job centre during your period on sick leave?

2 Did the services offered by the job centre meet your requirements and if so how?

3 In which way could your relatives participate in services offered by the job centre concerning your condition?

4 Psychoeducation is a voluntary offer among job-centre standard services, why did you choose to participate?

$5 \quad$ What has been your own initiative to improve recovery and to return to work?

$6 \quad$ Can you describe something that you have learned during your sick leave?

Only for informants offered the psychoeducation intervention

6 How did you experience participation in the psychoeducation?

7 What do you think about the content of the psychoeducation sessions?

Did any of your relatives participate in the psychoeducation?

8 And what effect did this have?

9 Can you describe something that you have learned from the psychoeducation? 


\section{Reflexivity}

The individuals constituted a vulnerable group, which was taken into consideration during all study phases by the adjustment of activities and questions in accordance with individual vulnerability. To obtain an understanding of the content and context, we participated in the psychoeducation sessions.

\section{Ethical considerations}

The informants were given oral and written information on the purpose of the interview. They were informed that their participation was voluntary and that they were free to end their participation at any time during the study. They were assured confidentiality and that the data would be securely stored. Each informant gave oral and written informed consent. Respect and consideration of the informants' personal stories were shown at all times. The study as well as the transfer of the informants' contact information from the research project have been approved by the Danish Data Protection Agency. According to The Danish Ethical Committee, neither the RCT nor the qualitative study needed to be registered (written communication, 9 July 2012).

\section{Results}

Four themes and six subthemes emerged from the interviews, reflecting the informants' experiences of psychoeducation in relation to the job centres' standard services (Table 3). The four themes were 'Access and relevance of psychoeducation in relation to the job centres' standard services', 'Social support', 'Self-care', and 'Psychoeducation intervention'.

\section{Table 3: Themes and subthemes generated from the interviews}

Access and relevance of psychoeducation and job-centre standard services

- Job-centre standard services

- Psychoeducation

Social support

- Including relatives in professional settings

- Seeking connection

\section{Self-care}

- Own initiative to recover

- Personal development

Psychoeducation intervention

- Content of sessions

- Time and atmosphere 


\section{Access and relevance of psychoeducation in relation to the job centres' standard services}

\section{Job-centre standard services}

All 16 informants described quite different access to the job centres' standard services. This divided the informants into three rather evenly sized groups: informants who were offered and participated in the job centres' standard services, informants who were offered but decided not to participate in the job centres' standard services, and informants who had not received any of the job centres' standard services.

Informants who received and participated in the job centres' standard services described both individual and group-based services. The majority of individual services consisted of a slow return to work and job training. A slow return to work was highly valued by employees on sick leave because having some days off work became an acceptable possibility and this option lowered the pressure to perform. Group-based services consisted of physical exercise, mindfulness, special care, and short lectures. Several informants took advantage of more than one of these services. The general attitude of this group seemed to be one of having confidence in and appreciative towards participating in the job centres' standard services, although the overall experience was that job centre's standard services were not specifically targeted at individuals on sick leave due to mental-health problems. Three of the 16 informants had participated in the programme targeting individuals on sick leave due to mental-health problems. Sussie, who participated in the physical exercise, said:

\footnotetext{
I feel that this was not the right place for me to be speaking out, because I might come across them (the other participants of the group) at the supermarket the day after. I would have liked a little more intimacy, for instance, a group only for persons suffering from stress. That would have been better because then I could begin opening up. Sussie (C)
}

Informants who had been offered but decided not to participate in the job centres' standard services described a somewhat similar range of services as did the informants who had received and participated in them. For some, reaching the locations of the job centres' standard services by public transport took too much time or was too much of a challenge because of their mentalhealth problem. Many described that their current treatment was very timeand energy-consuming and consisted of several initiatives. The general opinion in this group was that treatment and self-management were the primary goals before returning to work. One felt that the job centre was not very helpful in her situation. She asked for professionals with particular knowledge of mental-health problems to advise her on what to do and how to reach goals. When Thomas was asked why he did not participate in the job centre's standard services he said:

All of it has to be considered in relation to reserves of energy... as well as financial reserves. I might end up using more effort just to get out of my flat than any benefit I got. Thomas (I)

Informants who had not received any standard services expressed that they were not or were poorly informed about the possibilities of participating in the job centres' standard services. Some would have liked to participate in 
mindfulness or physical exercise; others had given up, assuming a rather negative tone towards the job centres. Olivia had been on sick leave for eight months:

I didn't hear one word [from the job centre].... I didn't get any suggestions or feedback as to what we might do in order to move forward. Olivia (I)

A few informants in this group were uncertain whether they had been offered any of the job centres' standard services. Throughout the interviews, several informants related experiences with moderate or severe cognitive impairments, such as poor memory. Katrin describes how she experienced an interview in combination with her mental-health problem:

I would have liked if they [the social workers] had suggested coming to my place for the interview... With such a severe anxiety for leaving home as I had then, I must say that I got nothing, absolutely nothing, out of the first meeting. I didn't hear anything of what she was saying. In fact, I remember nothing of what I should have remembered. Katrin (C)

\section{Psychoeducation}

Psychoeducation was a volunteer supplementary offer among the job centres' standard services. Therefore, all 16 informants had made a deliberate personal choice about participation. Their decisions about participating were based on combinations of five main issues: a wish to receive help to learn about mental health, to receive exercises and tools, to get away from home, to meet like-minded people, and to involve relatives. The informants emphasized that psychoeducation targeted their particular problem and provided a place to meet like-minded people. The general attitude towards psychoeducation was very positive and expectant. Hanna said:

It sounded relevant to me, and I hadn't had any offer of services in that respect, and I had no idea that there was that kind of offer so I was actually very happy when I saw it and I thought 'please help me'. Hanna (I)

Owing to the randomized controlled design, several participants expressed an awareness of the possibility that they might not receive an offer to participate in the psychoeducation sessions. They expressed positive terms like "hope" and "lucky" several times when talking about the possibility of participating in psychoeducation, highlighting the need of being seen and helped by rehabilitation professionals.

Perhaps I was lucky to be part of this course. I could see that there was potential for self-development. I knew I had to go in this direction. I couldn't change the world, but I could change who I am. Anne (I)

In summary, accessibility and relevance of a job centres' standard services and psychoeducation seemed to have both primary and secondary significance for the informants. The primary significance was to receive services that would support them to become active in their recovery process. The secondary significance was to be seen and respected by the rehabilitation professionals as an individual on sick leave with a mental-health problem. In relation to the job centres' standard services, psychoeducation seemed to 
meet both primary and secondary needs better. This indicates a higher relevance of psychoeducation than the job centres' standard services.

\section{Social support}

\section{Including relatives in professional settings}

The social support and involvement of relatives in relation to informants' sick leave and mental-health problems were mentioned frequently. Most informants pointed out both good and problematic experiences with family members as a result of their mental-health problem. The psychoeducation offered an evening session with the possibility to bring relatives along. This was considered a very important opportunity. No informant reported that a job centre offered a standard service that involved obtaining information from relatives on mentalhealth problems in a professional setting. One informant was informed about the possibility to bring her teenage son to a meeting for children with depressed parents. lan did not join the psychoeducation sessions. He said:

My father doesn't understand anything... he really ought to be sitting there, hearing about it from the professionals, because they are the only people that he believes in. They can provide the facts. Ian (C)

Five of the eight interviewed informants included in the psychoeducation intervention each brought one or two relatives. Two participants did not have any relatives, and one spouse was unavailable on the particular day of the session. The outcome of the session with relatives was described very individually, depending on the relatives' previous knowledge. Several informants mentioned that the session had increased the relatives' understanding of their mental problem, including the legitimacy to say 'no' and to be in need of time. Others mentioned experiences with relatives in supportive episodes during times when they were unable to cope with everyday life. Olivia's husband joined the evening for relatives. She said about him:

He was deeply affected by how bad I really was. In fact, he was quite shocked. For the next couple of days he was definitely very different, I must say... I could easily feel that he gave it a lot of thought... actually... not to say that he ignored the problem before, but now he had really got it under his skin. Olivia (I)

\section{Seeking connection}

One of the core elements was the informants' need of connection with others in the same situation. Social interaction with like-minded people was offered to three individuals through the job centres' standard services and to all participants joining the psychoeducation sessions. Connection with others in the same situation was perceived as a means of obtaining peer understanding, talking about one's own situation, learning from others, and avoiding isolation. John said:

I've realized that I'm not alone in this situation. Actually lots of people have experienced something like this, and that has given me a boost, socially speaking. John (I) 
Both the job centres' standard offer of the possibility to meet like-minded people and the psychoeducation were carried out as a group-based, slowopen sessions. This meant no waiting time for eligible individuals on sick leave to begin the offer and psychoeducation sessions, but it also meant that number of participants could vary from session to session. Though the offer and psychoeducation sessions were described as providing a comfortable and confident setting during a vulnerable period, the informants generally agreed and wished for one steady group instead of a slow-open group. They believed that at steady group would result in more social interaction, more sharing of experiences with other participants, and a greater feeling of inclusion, reassurance, and confidence. Chris said:

It would have been nice if it had been a group starting and ending together. Then perhaps it could have been possible, if others were interested, to meet afterwards, just for a cup of coffee at someone's house, having that possibility to be able to support each other because we were stuck in the same situation. Chris (I)

In summary, involving relatives in professional settings and providing them with information about mental-health problems seemed to increase social support to the individuals on sick leave. Activities offered in steady group sessions instead of slow-open groups may increase the experienced connection, social interaction, and feelings of reassurance. These elements may be interpreted as contributive to recovery. In relation to the job centres' standard services, the psychoeducation sessions seem to improve the possibility of social support.

\section{Self-care}

\section{One's own initiatives to recover}

The informants participated actively in their own recovery process. Most of the informants' own initiatives to recover involved treatment or preparing a way back to work. The initiatives towards treatment included all kinds of self-paid therapy, alternative treatment, physical exercise, diet, and voluntary counselling. Initiatives to prepare a way back to work were concerned with keeping contact with the boss or the colleagues and visiting the workplace. Many informants characterized these initiatives as really difficult and frightening tasks. Sussie was a registered childminder, working from home but with colleagues in the neighbourhood, and she said:

I was almost ready to go back to work, but then I asked if I could take another round with them all [my colleagues] ... that would become part of my self-care, because I had been afraid of bumping into them imagining my seeing them at the supermarket. I had been forced to go shopping somewhere else, because I was so shy or embarrassed about it all.... The fact that I met with them, face to face, and was able to talk to them about things that didn't concern my disease, that was really a supplement to my working with myself - which is part of it all.... My heart was really beating like crazy every time I went to see them, because I felt so embarrassed. I would rather be reported sick with a broken leg, because this was difficult to put into words. What if I had started crying? But they were so happy to see me.... I felt that they looked at me with different eyes. I had been so disillusioned. Sussie (C) 
Like Sussie, other informants who had returned to work at the time of the interview emphasized the importance of being encouraged to keep contact with the workplace during the sick-leave period, and they asked for more information about how to cope during their return to work. Anne attended the psychoeducation sessions and expressed:

\begin{abstract}
I believe that many people expect to be fully recovered when they start working again. They won't be. I know damned well that I won't be fit until two or three years after this ... it will take an incredibly long time. ... for me it is a matter of using tools like meditation, exercising, and the right food in order to manage the problems of everyday life and work. I have to take small breaks every second hour. There are things that I simply must stay away from doing because they are too much for me. Anne (I)
\end{abstract}

\title{
Personal development
}

Most informants were able to describe something which had changed or something they had learned about themselves during their sick leave. There were two types of changes. One was practical changes, like beginning to take a driver's licence, going back to study or changing treatment. The other type was broader personal changes, like the one Hanna related:

I became much better at accepting that depression was a disease - I have had this bad feeling wondering why I didn't pull myself together... but then I realized that you just can't. I would have been in a bad situation without this psychoeducation. Hanna (I)

Informants who participated in the psychoeducation sessions emphasized that they had become more conscious about themselves and their own situations. They frequently mentioned saying 'no', knowing themselves and their body better, making new strategies and reassessing their lives. Thomas put it this way:

I became very much aware that I'm the one responsible for my sick leave, with the choices I make and in terms of making a decision, and I try to handle my sick leave on my own terms. Thomas (I)

One expressed that the psychoeducation sessions had contributed to recovery and improvement but that the course alone was not enough.

In summary, informants' own initiatives to recover were considered necessary for some of them, since neither the job centres' standard services nor the psychoeducation sessions seemed to supply adequate information on how to cope with the workplace when on sick leave due to with a mental-health problem, or how to take care of one's self and to cope after returning to work. These elements might, however, be important for recovery. In relation to the job centres' standard services, the psychoeducation sessions seemed to present an increased possibility for broader personal development. 


\section{Psychoeducation intervention}

\section{Content of sessions}

The eight informants receiving the psychoeducation intervention described various perspectives on the session content. Five participants expressed that they had received new and important knowledge. Three already knew much of the information provided, which led to a disapproving view on the content of the sessions. Three wished for more individual counselling devoted to personal issues, but the common approach among the participants was an urge to develop, learn, and improve in order to make a change in their own situation. Thomas said:

I think it was nice to get a brush up, and I could easily see myself in all the points they mentioned, both in terms of when it went wrong and all those things. I could easily see where I went wrong. Thomas (I)

All eight informants emphasized the importance of the session in which a woman previously on sick leave presented her personal story in which she related the long process that was involving in overcoming and recovering from the manifold challenges presented by her mental problem. The majority of the informants expressed how they personally could relate to the experiences, reactions, patterns, and feelings in her sick-leave story

She, the one who had been sick herself, she gave us a lot, I think. There were so many similarities, and I could understand from what she told us that she had been ever sicker than me. ... Still, there were many things that I recognized, among others, that she had been angry with her husband, too. Olivia (I)

The teaching style was described as good, welcoming, and open, but too much information was provided by the structured slides. This form of instruction generated an experience of the sessions being rather inactive. Practical tools and exercises were given particularly high priority, either for use at home or together with other psychoeducation participants during the sessions. Some suggested more tools concerning the following: exercises making you decide what to do about the situation today and tomorrow, preparing you for what happens when you return to work, and how you could take care of yourself when starting work again or how to make achievable agreements with yourself. Tools and exercises already included in the sessions were writing in a diary, using a calendar, exercising, and coping tools. The tools and exercises were considered a manageable and useful way to work with oneself and seemed to create a hope of moving forward within the process of preparing oneself to return to work, to learn new routines or to develop personally. As Anne said:

When a person is suffering from stress, it is difficult to manage big changes. ... But with one little change each week for six weeks - that makes six small changes - that'll really be a lot. Anne (I)

\section{Time and atmosphere}

The psychoeducation sessions were offered within the first two months of a sick leave. A large number of the informants expressed difficulties remembering and taking in information during the early stages of sick leave. 
Some felt tired out after taking part in a two-hour session; others suggested half an hour break between the two hours. As Thomas said:

At the beginning I couldn't grasp much - two hours were just fine. I was completely filled up, I would say. I can take much more now than I could then. Thomas (I)

Generally, the psychoeducation participants felt that they were positively and openly met by the teachers and given room to ask and speak. If one had a bad day, it was acceptable not to contribute to the session. This created an accommodating atmosphere, which was emphasized as important for the motivation to show up every week. As Hanna said:

\begin{abstract}
I felt safe, felt that it was OK to turn up. I didn't feel any anxiety, thinking beforehand: oh no, this doesn't work... I just felt relaxed and I felt like getting some sort of.... don't know if I could call it confidence. At any rate I was not afraid to participate or to come forward with something. It was a nice atmosphere where I could easily say what I felt like saying. Hanna (I)
\end{abstract}

In summary, the advantages of the psychoeducation sessions are interpreted as a safe and accommodating environment with room for expression of personal stories. The sessions could be improved by introducing more tools and exercises and less information based on slides. To what degree the individual level of previous personal knowledge about mental-health problems affected the participants' views on the quality of the psychoeducation sessions is difficult to discern.

\title{
Discussion
}

The aim of the present study has been to explore experiences with psychoeducation both in relation to the standard services of a municipal jobcentre service from the perspective of individuals on sick leave and with regard to the relevance of the intervention and the elements that might contribute to recovery and improvement of psychoeducation as an intervention.

The results of the study showed that the rehabilitative process of returning to work for sick-listed individuals who are at risk of having a mental disorder was that the informants were to a high extent concerned about attaining the support needed to focus on mental-health problems. This was critical for them to become active in their own recovery process as well as being seen and respected by the rehabilitation professionals. Klanghed et al. (2004) have found that respectful and supportive interaction between rehabilitation professionals and sick-listed individuals was a positive encounter with regard to returning to work. The present study shows that psychoeducation offered a support that was considered relevant for individuals on sick leave who are at risk of having a mental disorder. The job centres' standard services were considered relevant to a lesser extent. This finding supports previously mentioned results from Hillborg (2010), which show that activities not relevant for individuals on sick leave with mental-health problems can lead to despondency and possible setbacks in the rehabilitation process. Furthermore, the elements that appear to contribute to recovery are social support through involvement of relatives and peers, self-development through 
information, practical tools and exercises, and an accommodating atmosphere. The positive influence of social support on recovery from mentalhealth problems has been emphasized in other studies (Grav, Hellzèn, Romild, \& Stordal, 2012; Maulik, Eaton, \& Bradshaw, 2010). The psychoeducation intervention also offered information on mental-health issues and seemed to have positive effects on coping, activation, and social interaction. The informants stressed the importance of sharing in groups, which is in line with Stjernswärd et al. (2013), who found that group-based interventions with peers are a source of support and inspiration and they increase the motivation to participate. The identified elements that might contribute to recovery were all present in psychoeducation. Most of a job centre's standard services did not include any of these elements. Psychoeducation appears to be a valuable initiative in individuals on sick leave due to mental problems, but could be a more effective tool if participating groups did not change during the therapy, there were more exercises, increased possibilities of sharing experiences with people in the same situation, and more information was given about how to cope after returning to work.

This qualitative study has contributed to a deeper understanding of the participants' perspective in relation to the RCT study. However, one of the limitations of this study was the small sample size, which was an analysis according to gender and age was not performed. In future studies we would recommend focus group interviews with a larger number of participants in order to be able to discuss relevant topics on the development of psychoeducation as part of return-to-work rehabilitation.

\section{Conclusion}

The results give insight into the experiences of individuals on sick leave due to mental problems who participate in return-to-work interventions in municipal job centres in Denmark with regard to the relevance of the initiatives, elements that might contribute to recovery, and improvements of psychoeducation as an intervention. Four themes emerged from the individual interviews that reflected the informants' experiences of psychoeducation and the job centres' standard services: 'Access and relevance of psychoeducation in relation to the job centres' standard services', 'Social support', 'Self-care', and 'Psychoeducation intervention'. The results highlight the importance of involvement of relatives in psychoeducation. Moreover, participating in the groups provided an access to social contact with people in the same situation. The informants participating in psychoeducation also requested tools and exercises to perform, such as homework. All informants emphasized the importance of initiatives on information on how to recover and cope while on sick leave.

\section{Acknowledgements}

We would like to thank all the informants and the four job centres for participating in the study. The study was funded by Tryg Fonden. 


\section{References}

Aho-Mustonen, K., Miettinen, R., \& Räty, H. (2010). Motives for participation, initial expectations, and satisfaction with group psychoeducation among forensic patients with schizophrenia. International Journal of Forensic Mental Health, 9(3), 226-236. doi:10.1080/14999013.2010.517257

Andersen, M. F., Nielsen, K. M., \& Brinkmann, S. (2012). Meta-synthesis of qualitative research on return to work among employees with common mental disorders. Scandinavian Journal of Work, Environment \& Health, 38(2), 93.

Arends, I., Bruinvels, D. J., Rebergen, D. S., Nieuwenhuijsen, K., Madan, I., NeumeyerGromen, A., . . Verbeek, J. H. (2012). Interventions to facilitate return to work in adults with adjustment disorders. The Cochrane Database of Systematic Reviews, 12, CD006389. doi:10.1002/14651858.CD006389.pub2; 10.1002/14651858.CD006389.pub2

Casañas, R., Catalan, R., del Val, J. L., Real, J., Valero, S., \& Casas, M. (2012). Effectiveness of a psycho-educational group program for major depression in primary care: A randomized controlled trial. BMC Psychiatry, 12, 230-244X-12230. doi:10.1186/1471-244X-12-230; 10.1186/1471-244X-12-230

Colom, F., Vieta, E., Sanchez-Moreno, J., Palomino-Otiniano, R., Reinares, M., Goikolea, J. M., . . . Martinez-Aran, A. (2009). Group psychoeducation for stabilised bipolar disorders: 5-year outcome of a randomised clinical trial. The British Journal of Psychiatry, 194(3), 260. doi:10.1192/bjp.bp.107.040485

Colom, F. (2011). Keeping therapies simple: Psychoeducation in the prevention of relapse in affective disorders. The British Journal of Psychiatry, 198(5), 338. doi:10.1192/bjp.bp.110.090209

Danish Employers' Confederation. (2014). Førtidspension og fleksjob, [Disability pension and flexicurity], The Danish Employers' Confederation, February 2012

Donker, T., Griffiths, K. M., Cuijpers, P., \& Christensen, H. (2009). Psychoeducation for depression, anxiety and psychological distress: A meta-analysis. BMC Medicine, 7(1), 79. doi:10.1186/1741-7015-7-79

Grav, S., Hellzèn, O., Romild, U., \& Stordal, E. (2012). Association between social support and depression in the general population: The HUNT study, a crosssectional survey. Journal of Clinical Nursing, 21(1-2), 111. doi:10.1111/j.13652702.2011.03868.x

Hillborg, H. (2010). Towards a working life? experiences in a rehabilitation process for people with psychiatric disabilities. Scandinavian Journal of Occupational Therapy, 17(2), 149. doi:10.3109/11038120902906303

Klanghed, U., Svensson, T., \& Alexanderson, K. (2004). Positive encounters with rehabilitation professionals reported by persons with experience of sickness absence. Work, 22(3), 247.

Malterud, K. (2011). Kvalitative metoder i medisinsk forskning: En innføring [Qualitative methods in medical research: an introduction] (3rd ed.). Oslo: Universitetsforlaget.

Marselisborgcentret, \& Rehabiliteringsforum Danmark. (2004). Rehabilitering i Danmark: Hvidbog om rehabiliteringsbegrebet [Rehabilitation in Denmark: White Paper on the concept of rehabilitation]. Århus: Marselisborgcentret.

Maulik, P. K., Eaton, W. W., \& Bradshaw, C. P. (2010). The effect of social networks and social support on common mental disorders following specific life events. 
Acta Psychiatrica Scandinavica, 122(2), 118 doi:10.1111/j.16000447.2009.1511.x

Morokuma, I., Shimodera, S., Fujita, H., Hashizume, H., Kamimura, N., Kawamura, A., . . Inoue, S. (2013). Psychoeducation for major depressive disorders: A randomised controlled trial. Psychiatry Research, 210(1), 134.

Muijzer, A., Brouwer, S., Geertzen, J. H., \& Groothoff, J. W. (2012). Exploring factors relevant in the assessment of the return-to-work process of employees on longterm sickness absence due to a depressive disorder: A focus group study. BMC Public Health, 12(1), 103. doi:10.1186/1471-2458-12-103

Nieuwenhuijsen, K., Bultmann, U., Neumeyer-Gromen, A., Verhoeven, A. C., Verbeek, J. H., \& van der Feltz-Cornelis, C. M. (2008). Interventions to improve occupational health in depressed people. The Cochrane Database of $\begin{array}{llll}\text { Systematic Reviews, (2):CD006237. doi(2), CD006237. } & \text { (2) }\end{array}$ doi:10.1002/14651858.CD006237.pub2; 10.1002/14651858.CD006237.pub2

Noordik, E. (2011). Exploring the return-to-work process for workers partially returned to work and partially on long-term sick leave due to common mental disorders: A qualitative study. Disability and Rehabilitation, 33(17-18), 1625. doi:10.3109/09638288.2010.541547

O'Connor, C., Gordon, O., Graham, M., Kelly, F., \& O'Grady-Walshe, A. (2008). Service user perspectives of a psychoeducation group for individuals with a diagnosis of bipolar disorder: A qualitative study. The Journal of Nervous and Mental Disease, 196(7), 568. doi:10.1097/NMD.0b013e31817d0193

Pedersen, P., Sogaard, H. J., Yde, B. F., Labriola, M., Nohr, E. A., \& Jensen, C. (2014). Psychoeducation to facilitate return to work in individuals on sick leave and at risk of having a mental disorder: Protocol of a randomised controlled trial. BMC Public Health, 14(1), 1288-2458-14-1288. doi:10.1186/1471-2458-14-1288

Rouget, B. W., \& Aubry, J. (2007). Efficacy of psychoeducational approaches on bipolar disorders: A review of the literature. Journal of Affective Disorders, 98(1), 11. doi:10.1016/j.jad.2006.07.016

Søgaard, H. J., \& Bech, P. (2009). Psychiatric disorders in long-term sickness absence -- a population-based cross-sectional study. Scandinavian Journal of Public Health, 37(7), 682. doi:10.1177/1403494809344357

Stafford, N., \& Colom, F. (2013). Purpose and effectiveness of psychoeducation in patients with bipolar disorder in a bipolar clinic setting. Acta Psychiatrica Scandinavica.Supplementum, (442), 11.

Ståhl, C., \& Edvardsson Stiwne, E. (2014). Narratives of sick leave, return to work and job mobility for people with common mental disorders in Sweden. Journal of Occupational Rehabilitation, 24(3), 543. doi:10.1007/s10926-013-9480-7

Stjernswärd, S., Bernce, R., \& Östman, M. (2013). 'Young women': The meaning of a collaborative program supporting young women's rehabilitation and reintegration into the labor market. Social Work in Public Health, 28(7), 672-84. doi: 10.1080/19371918.2011.593465. 